\title{
Raking and Relabeling for Imbalanced Data
}

This paper was downloaded from TechRxiv (https://www.techrxiv.org).

\section{LICENSE}

CC BY 4.0

SUBMISSION DATE / POSTED DATE

02-01-2022 / 05-01-2022

\section{CITATION}

Park, Seunghwan; Lee, Hae-Wwan; Im, Jongho (2022): Raking and Relabeling for Imbalanced Data. TechRxiv. Preprint. https://doi.org/10.36227/techrxiv.17712122.v1

$\mathrm{DOI}$ 


\title{
Raking and Relabeling for Imbalanced Data
}

\author{
Seunghwan Park, Hae-Hwan Lee, and Jongho Im*
}

\begin{abstract}
We consider the binary classification of imbalanced data. A dataset is imbalanced if the proportion of classes are heavily skewed. Imbalanced data classification is often challengeable, especially for high-dimensional data, because unequal classes deteriorate classifier performance. Undersampling the majority class or oversampling the minority class are popular methods to construct balanced samples, facilitating classification performance improvement. However, many existing sampling methods cannot be easily extended to high-dimensional data and mixed data, including categorical variables, because they often require approximating the attribute distributions, which becomes another critical issue. In this paper, we propose a new sampling strategy employing raking and relabeling procedures, such that the attribute values of the majority class are imputed for the values of the minority class in the construction of balanced samples. The proposed algorithms produce comparable performance as existing popular methods but are more flexible regarding the data shape and attribute size. The sampling algorithm is attractive in practice, considering that it does not require density estimation for synthetic data generation in oversampling and is not bothered by mixed-type variables. In addition, the proposed sampling strategy is robust to classifiers in the sense that classification performance is not sensitive to choosing the classifiers.
\end{abstract}

Index Terms—calibration, classification, mixed-type, oversampling, undersampling.

\section{Introduction}

$\mathrm{C}$ LASS imbalance problems occur when the majority class instances outnumber the minority class instances. In recent years, this class imbalance problem has drawn significant interest in many real-world classification problems (e.g., fraud detection in bank account transactions or telephone calls, biomedical diagnosis, text classification, information retrieval and filtering, and college student retention [1], [2]).

The objective of the traditional classifier is to optimize accuracy for the whole dataset. However, in the imbalanced data problem, the classifier tends to favor the majority class, resulting in a significant classification error over the minority class. In particular, a random classifier that predicts all instances as the majority class can achieve very high accuracy despite misclassifying all minority instances [3]. This misclassifying of the minority class can result in high costs, as failing to identify the minority class instances could cause enormous losses. Thus, the classifier in the imbalanced problem is required to reduce its classification error for the minority and majority classes.

Given the prevalent problems of data imbalance, several approaches have been suggested to improve the accuracy of classification models. Despite the understanding that no single approach can address all problems of imbalanced data, studies have often highlighted the usefulness of sampling methods as a preliminary step to balancing the class distribution [2], [4], [5]. In this research, we focus on sampling methods to handle highly imbalanced data in the binary classification.

The most popular oversampling method is the synthetic minority oversampling technique (SMOTE) proposed by [6]. Additionally, SMOTE generates extra synthetic minority data

- S. Park is with the Department Department of Information and Statistics, Kangwon National University, Republic of Korea. E-mail: stat.shpark@kangwon.ac.kr

- H. Lee, and J. Im are with the Department of Statistics 8 Data Science, Yonsei University, Seoul, 03722, Republic of Korea. E-mail: $\{0210 h w a n, i j h 38\} @ y o n s e i . a c . k r$

*: correponding author points by interpolating the spaces between existing minority data points. Since SMOTE, many other variations of synthetic oversampling have been proposed and have proved to be powerful for handling the data imbalance problem for classification. In addition, [7] provides essential characteristics of the extensions of SMOTE based on seven aspects: (i) initial selection of instances to be oversampled, (ii) integration with undersampling as a step in the technique, (iii) type of interpolation, (iv) operation with dimensionality changes, (v) adaptive generation of synthetic examples, (vi) relabeling, and (vii) filtering of noisy generated instances. The most frequent properties exploited by synthetic oversampling methods are initial selection and adaptive generation of synthetic examples.

The adaptive synthetic sampling technique (ADASYN) [8] automatically determines the number of synthetic instances to be created based on the density distribution of the minority class. Agglomerative hierarchical clustering [9] uses $K$-means clustering algorithm to generate synthetic samples to balance the data. Density-based SMOTE employs a density-based clustering approach and generates synthetic samples along the shortest path from each minority sample to the pseudo-center of a minority class cluster. In contrast, borderline-SMOTE [10] categorizes the minority instances based on the majority neighbors and samples from instances closer to the separating boundary. The majority weighted minority oversampling technique (MWMOTE) [4] analyzes the most challenging minority examples and assigns each a weight according to the Euclidean distance from the nearest majority examples. Random oversampling method, named by ROSE [11], is established by generating new artificial data from classes according to the smoothed bootstrap form and kernel density estimation. Another variation of SMOTE, safe-level SMOTE [12], focuses on developing a method to suppress the harmful effects of the synthetic minority sample and only generates safe ones.

There exist other oversampling approaches which do not generate synthetic samples in the minority class. For example, SPY [13] and moving to adaptive samples in imbalanced 
datasets (MASI) [14] employ the relabeling technique during synthetic generation. The relabeling technique rebalances class distribution by changing the labels of some majority class samples to those of minority class samples. These majority samples, whose labels are changed, are close to the borderline of the majority class and nearest neighbor samples located near the minority class [10].

However, these variations of SMOTE do not perform well for all problems because of the dataset complexity issues using heuristic rules. In particular, some research [10], [15] has suggested that these methods cannot improve or reduce classification performance. We provide empirical evidence that these synthetic oversampling methods become inappropriate and fail to generate useful synthetic minority instances for real-world imbalanced classification problems with some or all of the categorical feature variables.

In this paper, we propose a novel resampling approach combining raking and relabeling $(\mathrm{R} \& \mathrm{R})$ techniques as an alternative method to construct balanced samples. The main idea of the R\&R method is to generate additional minority class instances from the majority class through raking, resampling, and relabeling procedures. As the proposed method does not generate and use synthetic data, it is directly applicable to imbalanced datasets with all categorical feature variables. Extensive benchmark tests comparing the proposed $\mathrm{R} \& \mathrm{R}$ to other popular oversampling methods are also presented. Three types of imbalanced datasets are considered: i) all continuous feature variables, ii) all categorical feature variables, and iii) mixed continuous and categorical feature variables. In imbalanced datasets with all categorical and mixed feature variables, $\mathrm{R} \& \mathrm{R}$ outperforms existing wellknown oversampling methods for the geometric mean $(G-$ mean), $F_{1}$ score, and area under the curve (AUC) as evaluation criteria. Moreover, $\mathrm{R} \& \mathrm{R}$ is more robust, which means the performance criterion value of the proposed method is less sensitive across several different scenarios.

The rest of this paper is organized as follows. First, we provide a brief review of the existing research developments targeting the imbalanced data problem in Section 2. In Section 3 , we proposed the $R \& R$ method for highly imbalanced data and describe its various components in details. The data descriptions and assessment metrics for learning algorithms are described in Section 4. In Section 5, we present the experimental study and simulation results to compare and evaluate the performance of various learning algorithms. Finally, the conclusion is provided in Section 6.

\section{Related Works}

Numerous methods have been developed to handle imbalance problems and improve the accuracy of the classification models. Generally, most of these fall into three categories: algorithm-based, cost-sensitive, and sampling-based methods [1], [16]. Algorithm-based methods directly modify the existing classifier learning algorithm to alleviate its bias toward the majority class [17]. Cost-sensitive methods assign a higher cost to the misclassification of minority class instances [18]. Sampling-based methods address the imbalance problem by modifying datasets to more balanced samples before training the classifier [3], [10].

Although no single state-of-the-art approach works well with all imbalanced data, many studies have demonstrated that sampling methods to balance the class distribution at a preliminary step are a useful solution [2], [4], [5], [7]. We focus on a review of the methods developed with a sampling approach.

Sampling methods modify the data to a more balanced distribution by either undersampling majority samples or oversampling minority samples. A simple sampling strategy is to use the undersampling or oversampling method. Undersampling methods create a subset of the original dataset by eliminating examples. This discarding can be random, which is called random undersampling. In contrast, it can be performed using statistical knowledge, which is called informed undersampling. Undersampling methods are less efficient than oversampling methods because undersampling induces the loss of important information in the original data for classification. Oversampling methods create a superset of the original dataset by adding minority class samples to an imbalanced dataset. The simplest oversampling method is random oversampling, which randomly duplicates minority samples to the desired size. Random oversampling can increase the chances of overfitting because it makes exact copies of the original data.

Synthetic oversampling methods generate synthetic minority class samples to balance the class distribution. In addition, [6] proposed SMOTE, where new minority samples are created between randomly selected minority samples and their $\mathrm{k}$-nearest neighbors (KNN) using the random interpolation of both instances. However, it would be meaningless to classify linear interpolate instances between two samples with little contribution to the decision boundary. Various extensions of SMOTE have been proposed to address this limitation. We provide a brief review of the most well-known oversampling methods considered in the numerical experiments to analyze the performance of the proposed method. A detailed review of all other synthetic techniques was given by [7].

Borderline-SMOTE [10] is based on the finding that most borderline and nearby samples are more easily misclassified than those far from the borderline. Thus, the method identifies samples belonging to the borderline using the ratio between the majority and minority examples in the neighborhood of each oversampled instance. For example, noisy samples (including all neighbors from the majority class) are not considered in the learning process.

Further, ADASYN [8] employs a weighted distribution depending on the type of minority examples according to their complexity for learning. The number of synthetic instances is created based on the level of difficulty of each minority example. The level of difficulty depends on the number of samples belonging to the majority class in the neighborhood of the minority samples.

Moreover, MWMOTE [4] draws from the assumption that other oversampling methods may generate the wrong synthetic minority samples. This method analyzes hard-to-learn minority class samples and assigns each a weight according to the Euclidean distance from the nearest majority examples. The samples closer to the decision boundary and the samples of small clusters are assigned higher weights than others. The synthetic examples are generated from the weighted informative minority class instance using a clustering approach, ensuring that they must lie inside a minority class cluster. One of the limitations is that nominal features with MWMOTE 
were not initially considered.

Furthermore, ROSE [11] was established by generating new artificial data from classes using a multivariate kernel density estimation and smoothed bootstrap resampling technique. It takes advantage of the kernel density estimation to maintain the distribution of the feature variables in each class but cannot be applied to imbalanced data with categorical feature variables.

\section{Raking, Resampling, and Relabeling}

Let $y$ be a binary response that has two distinct values $\{0,1\}$, and $\boldsymbol{x}$ denotes $p$-dimensional feature variables. Let $M_{0}=\left\{i ; y_{i}=0\right\}$ and $M_{1}=\left\{i ; y_{i}=1\right\}$ be the index sets of size $n_{0}$ and $n_{1}$, respectively. In this paper, we consider highly imbalanced data (i.e., $n_{0} \gg n_{1}$ ); thus, $M_{0}$ and $M_{1}$ represent the majority class and minority classes, respectively. Our goal is to construct balanced samples using resampling techniques, popularly used in survey statistics. We defined a target balanced sample size as $2 \times m$, and set $n_{0}^{b}=n_{1}^{b}=2 \times m$, where $n_{0}^{b}$ and $n_{1}^{b}$ are the sizes of the balanced samples for the majority and minority classes, respectively.

Figure 1 illustrates the resampling strategy, which employs $R \& R$ procedures. As the first step, we constructed raking weights so that the predetermined design variables have the same sample moments between majority and minority classes:

$$
\sum_{i \in M_{0}} w_{i}^{*} \boldsymbol{z}_{i}=n_{1}^{-1} \sum_{j \in M_{1}} \boldsymbol{z}_{j}
$$

where $w_{i}^{*}$ denotes the raking weight imposed only on the majority samples, and $z \equiv g(\boldsymbol{x}): \mathbb{R}^{p} \rightarrow \mathbb{R}^{q}$ represents a $q$-dimensional vector of design variables converted from the initial features. Once we obtained the raking weights for all cases in $M_{0}$, we resampled the majority cases of size $m$ using a probability proportional to the size sampling (PPS) [19] and relabeled the sampled majority cases as minority samples. Next, we generated $m$ bootstrap samples from the initial minority class using simple random sampling with replacement (SRSWR). We then obtained balanced minority samples, denoted by $M_{1}^{b}$, of size $n_{1}^{b}=2 \times m$, combining both resampled cases.

As the second step, we constructed the balanced majority samples with a size of $n_{1}^{b}=2 \times m$. We applied the same raking technique on the remaining initial majority samples of size $n_{0}^{b}-n_{1}^{* b}$, where $n_{1}^{* b}$ denotes the bootstrap samples relabeled for the new minority samples. First, we computed the raking weight $p_{i}^{*}$ using the initial majority class $M_{0}$ and the adjusted majority class $M_{0}^{*}$ :

$$
\sum_{i \in M_{0}^{*}} p_{i}^{*} \boldsymbol{z}_{i}=n_{0}^{-1} \sum_{j \in M_{0}} \boldsymbol{z}_{j},
$$

where $M_{0}^{*}$ indicates the initial majority samples, excluding relabeled samples. Once we obtained the raking weights $p_{i}^{*}$, we reconstructed bootstrap majority samples, denoted by $M_{0}^{b}$, with a size of $n_{0}^{b}$, which is the same size as the new minority samples.

Algorithm 1 describes R\&R algorithm. Details about the algorithm, including assumptions, design variables, raking, and resampling, are introduced in subsequent subsections. We used these samples to learn the classifiers after we constructed the balanced majority and minority samples, $M_{0}^{b}$ and $M_{1}^{b}$.

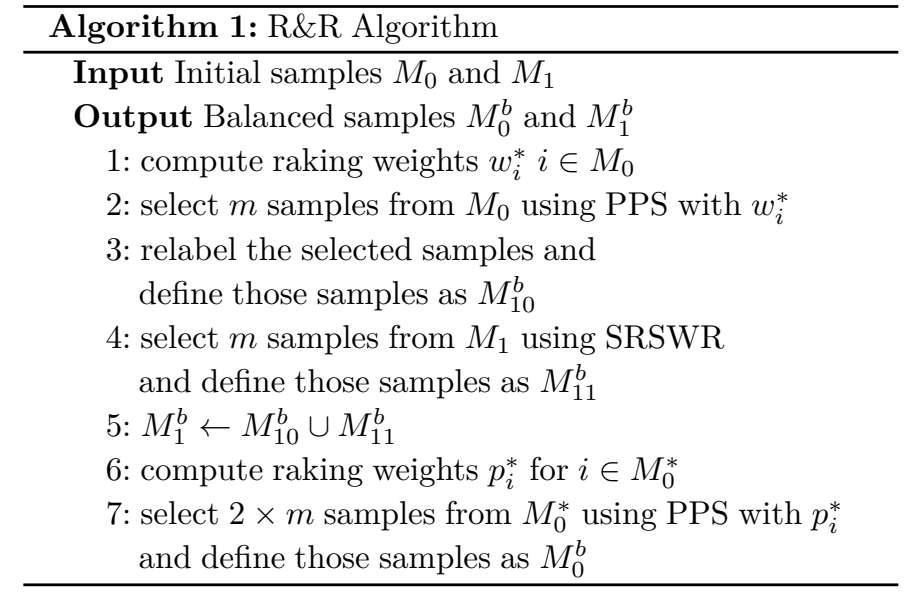

Remark 1. The proposed method implicitly requires the same support on both majority and minority classes, such that $0<P(y=1 \mid \boldsymbol{z})<1$, or equivalently,

$$
D(\boldsymbol{z})=\{\boldsymbol{z} ; f(\boldsymbol{z} \mid y=0)>0\}=\{\boldsymbol{z} ; f(\boldsymbol{z} \mid y=1)>0\} .
$$

This condition is natural because we relabel and use majority samples as the minority class. When the majority and minority classes have different support, it is easier to construct an effective classifier because the samples are separated on the nonoverlapped feature domains.

\subsection{Design Variables}

We employed design variables transformed from the feature variables $\boldsymbol{z} \equiv g(\boldsymbol{x}): \mathbb{R}^{p} \rightarrow \mathbb{R}^{q}$ with the known transformation function $g(\cdot)$. The number of design variables $q$ is not necessarily smaller than $p$. For example, with two dimensional features, denoted by $x_{1}$ and $x_{2}$, we can use three design variables, $x_{1}, x_{2}$ and $x_{1} x_{2}$. The raking weights, satisfying Eq. (1), make the weighted mean of $x_{1}, x_{2}$ and $x_{1} x_{2}$ of the majority samples equal to the averaged values of the minority samples, respectively.

We selected continuous design variables that have significant differences in the first moments between the majority and minority classes and chose categorical design variables that have significant differences in the distribution between the two classes for computational efficiency. Many statistical tests, such as the $t$-test for continuous variables or the $\chi^{2}$ test for categorical variables, can be conducted to determine whether a significant difference exists in the first moments or distributions between majority and minority classes.

Even when the marginal means or distributions of the design variable between the two classes are different, it is necessary to assume some overlap may exist between the data $\boldsymbol{x}$ where $y=1$ and the distribution of $\boldsymbol{x}$ given $y=0$. It could be that a design variable is an imbalanced binary variable with a value of 0 or 1 ; thus, the variable might never take the value of 1 in the minority class. In this case, $\overline{\boldsymbol{z}}$, the average of the $\boldsymbol{z}_{i}$ for which $y=1$, is right on the boundary of the support of the distribution $f(\boldsymbol{z} \mid y=0)$, and the raking weights in (1) might be substantial.

In addition, [20] employed the overlap condition that the relative interior of the convex hull of the $\boldsymbol{x}^{\prime} s$ for $y=0$ must intersects with that of $y=1$. Further, [21] also described the overlap condition using the following definition: 


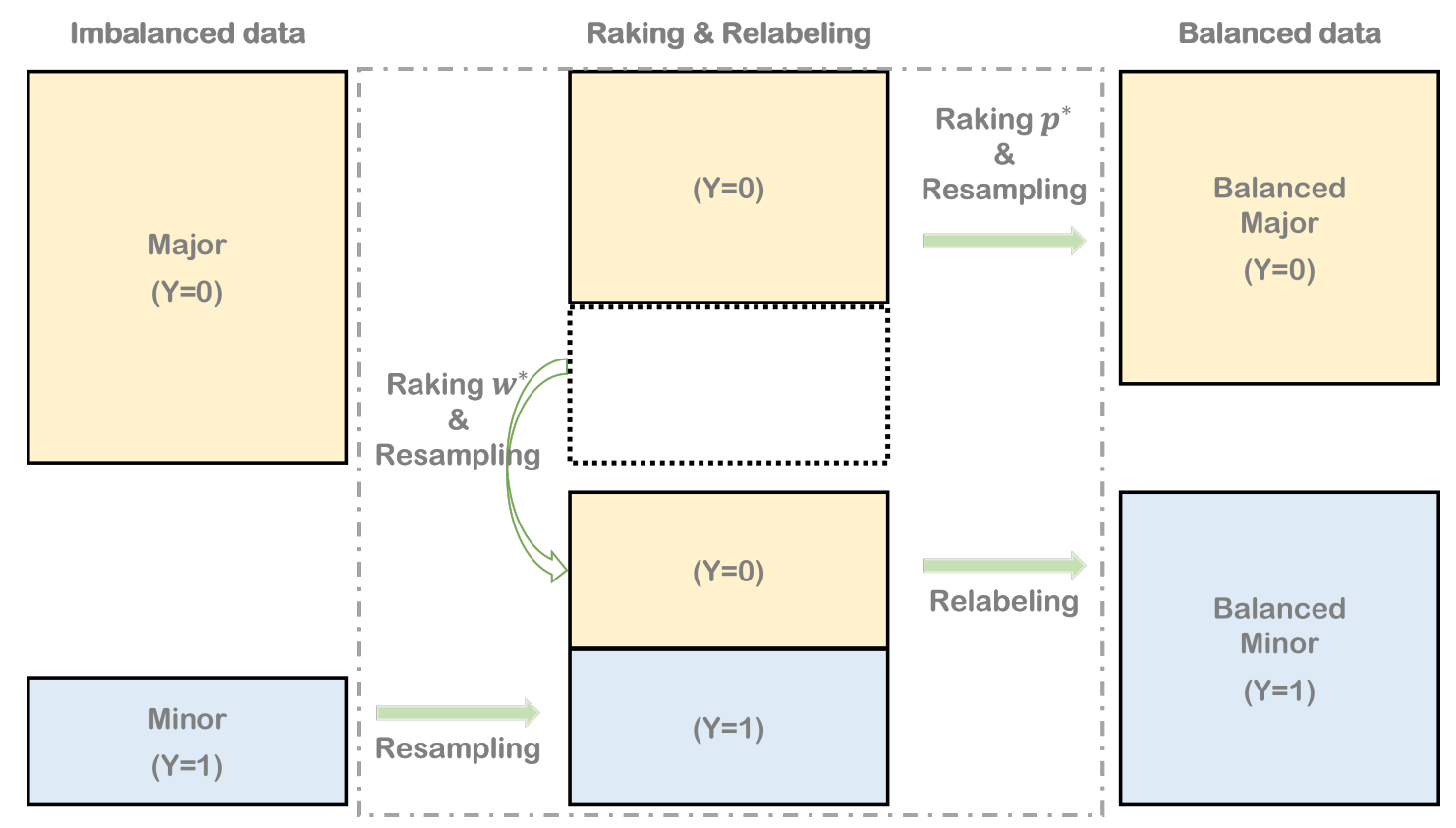

Fig. 1: Illustrative working frame of the proposed R\&R algorithm

Definition 1. The distribution $F$ on $\mathbb{R}^{d}$ has the point $x_{*}$ surrounded if

$$
\int_{\left(x-x_{*}\right)^{\prime} \omega>\epsilon} d F(x)>\delta
$$

holds for some $\epsilon>0$, some $\delta>0$ and all $\omega \in \Omega$, where $\Omega=\left\{\omega \in \mathbb{R}^{d} \mid \omega^{\prime} \omega=1\right\}$, the unit sphere in $\mathbb{R}^{d}$.

We use the overlapping index $\eta$ proposed by [22] to measure the distance between the two distributions. The overlap index $\eta$ computes the overlapping index $\eta: \mathbb{R}^{n} \times \mathbb{R}^{n} \rightarrow[0,1]$, which is defined as follows:

$$
\eta(A, B)=\int_{\mathbb{R}^{n}} \min \left\{f_{A}(x), f_{B}(x)\right\} d x,
$$

where $f_{A}(x)$ and $f_{B}(x)$ are two real probability density functions, and the integral can be replaced by summation in the discrete case.

Remark 2. In general, the overlapping index is normalized to vary between zero and one; thus, $\eta=1$ means that the two distributions are the same. In contrast, $\eta=0$ indicates that the two distributions are completely separate. The uncertainty of the $\eta$ index can be obtained using a bootstrap approach. The overlapping index can be easily computed using the R-package overlapping [22].

\subsection{Raking Weights}

We first describe how to obtain the raking weights $w_{i}^{*}$ used to generate the relabeled samples. To construct raking weights satisfying Eq. (1), we apply a calibration method [19], [23] on each design variable $z^{(j)}, j=1, \ldots, q$, where $z^{(j)}$ is the $j$ th element of $\mathbf{z}=\left(z^{(1)}, \ldots, z^{(q)}\right)$. In the case of continuous variable $z^{(j)}$, we can obtain the raking weights $w_{i}^{(j)}$ by maximizing the following scaled Shannon entropy $Q$,

$$
Q=-\sum_{i \in M_{0}} w_{i}^{(j)} \log w_{i}^{(j)} / d_{i}
$$

where $d_{i}$ denotes the initial weights with $\sum_{i} d_{i}=1$, while the raking constraint (1) holds. This constrained optimization problem is essentially equivalent to maximize the Lagrange function [24]:

$L=-\sum_{i \in M_{0}} w_{i}^{(j)} \log w_{i}^{(j)} / d_{i}+\left(n_{1}^{-1} \sum_{i \in M_{1}} \lambda^{T} \mathbf{z}_{i}^{(j)}-\sum_{i \in M_{0}} w_{i} \lambda^{T} \mathbf{z}_{i}^{(j)}\right)$,

where $\lambda$ is a $2 \times 1$ vector of Lagrange multipliers and $\mathbf{z}_{i}^{(j)}=$ $\left(1, z_{i}^{(j)}\right)$. The constant term is combined with the target design variable to enforce the sum of raking weights is equal to 1 (i.e., $\left.\sum_{i \in M_{0}} w_{i}^{(j)}=1\right)$. According to the Lagrange function in (4), the raking weights can be expressed as an exponential function such that

$$
w_{i}^{*(j)} \propto d_{i} \exp \left(-\lambda^{T} \boldsymbol{z}_{i}^{(j)}\right) .
$$

Thus, the constraint (1) can be written again, as follows:

$$
U(\lambda) \equiv \sum_{i \in M_{0}} d_{i} \exp \left(-\lambda^{T} \mathbf{z}_{i}^{(j)}\right) \mathbf{z}_{i}^{(j)}-n_{1}^{-1} \sum_{j \in M_{1}} \mathbf{z}_{j}^{(j)}=0 .
$$

We now obtain the raking weights by solving the estimating equation $U(\lambda)$ in (5) using conventional Newton-type algorithms, and the weights are automatically regularized so that its sum is equal to 1.

For discrete design variables, we can directly use a poststratification method [19], often used in survey sampling. To introduce the raking procedure, assume that the design variable $z^{(j)}$ has $K$ distinct values $\{1, \ldots, K\}$ and that the majority and minority samples are partitioned into $K$ subsets, respectively,

$$
M_{0 k}^{(j)}=\left\{i \in M_{0} \mid z_{i}^{(j)}=k\right\}, k=1, \ldots, K,
$$

and

$$
M_{1 k}^{(j)}=\left\{i \in M_{1} \mid z_{i}^{(j)}=k\right\}, k=1, \ldots, K .
$$

After we constructed the subsets $M_{0 k}^{(j)}$ and $M_{1 k}^{(j)}$, the raking weights for the $j$ th design variable $w_{i}^{*(j)}$ were computed as 
post-stratification weights such that

$$
w_{i}^{*(j)}=d_{i} \times \frac{n_{1 k}^{(j)} / n_{1}}{\sum_{s \in M_{0 k}^{(j)}} d_{s}} \quad i \in M_{1 k}^{(j)}
$$

where $d_{i}$ is the initial weight with $\sum_{i \in M_{0}} d_{i}=1$, and $n_{1 k}^{(j)}$ is the sample size of $M_{1 k}^{(j)}$. From its construction, we obtain

$$
\sum_{i \in M_{0 k}^{(j)}} w_{i}^{*(j)}=\frac{n_{1 k}^{(j)}}{n_{1}}, \quad \sum_{k=1}^{K} \sum_{i \in M_{0 k}^{(j)}} w_{i}^{*(j)}=1,
$$

which indicates that the raking weights $w_{i}^{*(j)}$ satisfy the constraint (1).

To obtain the final raking weights $w_{i}^{*}$, we sequentially and iteratively implemented the raking procedure for each design variable $\boldsymbol{z}^{(j)}, j=1, \ldots, q$, until the weighted means of design variables of the majority samples approach the average of design variables of the minority samples with a stop criterion. The proposed raking procedure for computing $w_{i}^{*}$ is introduced in Algorithm 2.

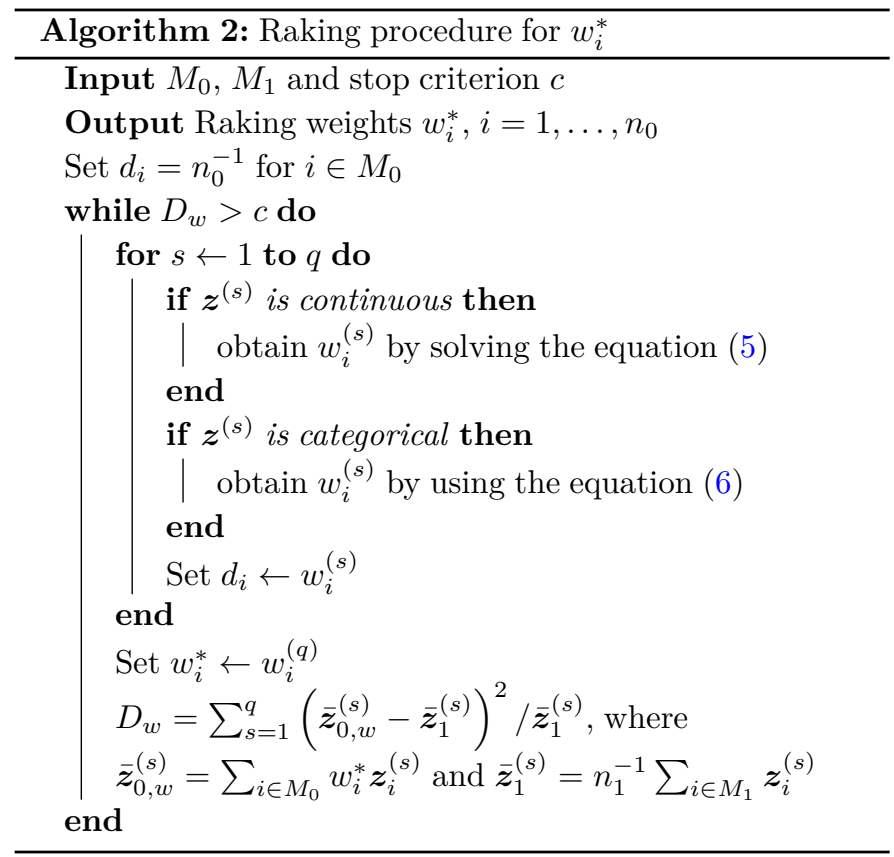

Another raking weights $p_{i}^{*}$ can be obtained using the same techniques presented above by replacing datasets from $M_{0}$ and $M_{1}$ with $M_{0}^{*}$ and $M_{0}$, respectively. Although we described the raking procedure marginally, these procedures can be applied to multiple design variables. However, it is challenging to preserve the joint structure of the design variables due to the sparsity problem. Algorithm 2 presents step-by-step raking procedure for obtaining the raking weights $w_{i}^{*}$ for $i \in M_{0}$ and $p_{j}^{*}$ for $j \in M_{0}^{*}$.

Remark 3. Because a raking procedure is sequentially applied to each design variable, the order of the raking process is often critical. Thus, $\boldsymbol{z}^{(q)}$ is more likely to have the same values between the weighted majority samples and averaged minority samples, rather than the first design variable $\boldsymbol{z}^{(1)}$. Therefore, we can strategically set the raking order of important features later.

\subsection{PPS Sampling}

Raking weights can be directly used for selecting samples using a multinomial distribution, because we allow replicated samples in balanced data. Let $\Delta_{i}, i=1, \ldots, M_{0}$, be the number of times majority sample $i$ is observed over the $m$ trials, then $\Delta=\left(\Delta_{1}, \ldots, \Delta_{n_{0}}\right)$ follows a multinomial distribution with the probability density function of

$$
\operatorname{Pr}\left(\Delta_{1}=\delta_{1}, \ldots, \Delta_{n 0}=\delta_{n_{0}}\right)=\frac{m !}{\delta_{1} ! \cdots \delta_{n 0} !} \prod_{i \in M_{0}}\left\{w_{i}^{*}\right\}^{\delta_{i}},
$$

where $\sum_{i \in M_{0}} \delta_{i}=m$ and $\sum_{i \in M_{0}} w_{i}^{*}=1$. Because each unit in the majority sample is selected with probabilities proportional to $w_{i}^{*}$, this sampling design is essentially a probability proportional to size (PPS) [19] sampling. In addition, we select the remained $m$ samples from the minority samples using SRSWR. Combining all generated samples, we can construct a balanced sample with $y_{i}=1$.

Similarly, we can generate another balanced sample for the majority class, $y_{j}=0, j=1, \ldots, 2 m$. We select $2 m$ samples from $M_{0}^{*}$ with the raking weights $p_{j}^{*}, j=1, \ldots, n_{0}-m$. Moreover, $D_{j}$ is defined as the number of times sample $j \in M_{0}^{*}$ is observed over the $2 m$ trials, and the random variable $D=\left(D_{1}, \ldots, D_{n_{0}^{*}}\right)$ becomes a multinomial distribution with the following probability density function:

$$
\operatorname{Pr}\left(D_{1}=d_{1}, \ldots, D_{n 0}=d_{n 0}\right)=\frac{(2 m) !}{\delta_{1} ! \cdots \delta_{n_{0}^{*}} !} \prod_{j \in M_{0}^{*}}\left\{p_{i}^{*}\right\}^{d_{j}},
$$

where $\sum_{j \in M_{0}^{*}} d_{j}=2 m$ and $\sum_{j \in M_{0}^{*}} p_{j}^{*}=1$.

\section{Experiments}

\subsection{Data}

To offer various domains in experiments, we chose 16 realworld datasets with different numbers of samples, variables, and varying imbalance ratios (IRs). These datasets originated from public sources: 1) Knowledge Extraction based on Evolutionary Learning data repository [25], 2) UCI machine learning repository [26], 3) HDDT collection [27] and 4) previous studies [28], [29], [30]. All these repositories contain an extended benchmark for the most imbalanced datasets in the literature.

Two criteria were considered when we select datasets. First, large-scale datasets were preferred because most previous experimental studies have not been considered on largescale datasets. Datasets containing more than 1,000 instances were ignored and not tested [31]. In the empirical study, among 16 real-world datasets, 10 datasets contain more than 1,000 instances, and four datasets contain more than 10,000 instances. Second, highly imbalanced datasets are the primary focus of the experiment. Therefore, we selected datasets with an IR higher than 9.

These datasets can be grouped into three categories: i) continuous datasets containing all continuous feature variables, ii) categorical datasets containing all categorical feature variables, and iii) mixed datasets with continuous and categorical feature variables. The data were summarized in Table 1 by listing the dataset name, number of observations, number of variables, and IR, where $V_{c}$ presents the number of continuous attributes and $V_{d}$ indicates the number of categorical variables. 
TABLE 1: Description of Datasets

\begin{tabular}{|c|c|c|c|}
\hline \multicolumn{4}{|l|}{ Categorical Datasets } \\
\hline Datasets & \#Instances & \#Atts & IR \\
\hline Boundary & 3,505 & 175 & 28 \\
CaM & 18,916 & 132 & 19 \\
Car good & 1,728 & 6 & 24 \\
Led & 443 & 7 & 11 \\
PhosS & 11,411 & 480 & 18 \\
\hline
\end{tabular}

\begin{tabular}{|c|c|c|c|}
\hline \multicolumn{2}{|l|}{ Continuous Datasets } \\
\hline Datasets & \#Instances & \#Atts & IR \\
\hline CompuStat & 13,657 & 20 & 25 \\
Glass4 & 214 & 9 & 15 \\
Mammography & 11,183 & 6 & 42 \\
Oil & 937 & 49 & 22 \\
Wine Quality & 656 & 11 & 35 \\
Yeast & 482 & 8 & 23 \\
\hline \multicolumn{3}{|l}{} \\
\hline
\end{tabular}

\begin{tabular}{|c|c|c|c|}
\hline \multicolumn{4}{|l|}{ Mixed Datasets } \\
\hline Datasets & \#Instances & \#Atts $\left(V_{c} / V_{d}\right)$ & IR \\
\hline Abalone & 2,338 & $8(7 / 1)$ & 39 \\
Flare-F & 1,066 & $11(7 / 4)$ & 24 \\
Hypo & 3,163 & $19(5 / 14)$ & 20 \\
Sick & 3,621 & $21(1 / 20)$ & 16 \\
Vowel0 & 988 & $12(10 / 2)$ & 10 \\
\hline
\end{tabular}

\subsection{Experimental Setup}

To analyze the performance of $\mathrm{R} \& \mathrm{R}$, we compared the proposed approach with five well-known methods of sampling methods: ROSE [11], SMOTE [6], ADASYN [8], MWMOTE [4], and conventional random over-sampling (ROS). Every method was set to generate an equal number of majority and minority class instances as the balanced samples. In addition, SMOTE, ADASYN, MWMOTE, and ROS were set to oversample the minority class until the quotient size of both classes reaches 1 , which makes the number of balanced datasets much larger than the original size, becoming larger with the increasing IR. On the other hand, ROSE oversamples minority instances and undersamples majority examples so that we obtain a balanced sample sizes between two classes. Similarly, R\&R also undersamples majority examples while relabeling the majority class instances to the minority class.

Each sampling algorithm has user-defined parameters. For SMOTE and ADASYN, five nearest neighbor values are used to generate balanced samples. Moreover, MWMOTE requires six parameters:

- $k_{1}$ for the Euclidean KNN to detect noisy minority samples,

- $k_{2}$ for the Euclidean KNN to determine the borderline majority class samples,

- $k_{3}$ for the Euclidean KNN to select minority samples,

- $c_{p}$ for the number of clusters, and

- $c_{f}(t h)$ and cmax, the parameters for smoothing and rescaling the values of different scaling factors used in weighting the minority class samples

These values are $k_{1}=5 \%, k_{2}=3 \%$, and $k_{3}=50 \%$ of the original minority class samples $c_{p}=3, c_{p}(t h)=5$, and cmax $=2$, respectively, from [4].

Some alternative methods could not handle nominal features. For example, SMOTE and MWMOTE algorithms were evaluated on the dataset where only continuous features were extracted to generate a new dataset [6], [4]. Therefore, to carry out a fair evaluation with other methods, categorical features were converted to dummy variables so that such information also could be used in every sampling method.

For classifiers, we chose the most widely used classifiers: i) support vector machine (SVM), ii) random forest (RF), and iii) naive Bayes (NB). The radial basis function (RBF) kernel was used for higher-dimensional kernel space for SVM classifiers, where the data are projected [32]. Like oversampling algorithms, $\mathrm{R}$ packages were applied to these classifiers, using randomForest [33] to implement RF and e1071 [34] for SVM and NB.

To obtain simulation results, a series of 100 trials were conducted for each dataset $X$. In each trial, the dataset $X$ was randomly divided into two equal sets $X_{\text {training }}$ and $X_{\text {test }}$, where the binary skewed class distribution of both sets was also the same. Then, the sampling algorithms are carried out to generate balanced datasets using $X_{\text {training }}$. After using the newly created set for training the learning algorithms, the resulting classifiers were tested on $X_{\text {test }}$. The whole experimental procedure is summarized at Algorithm 3.

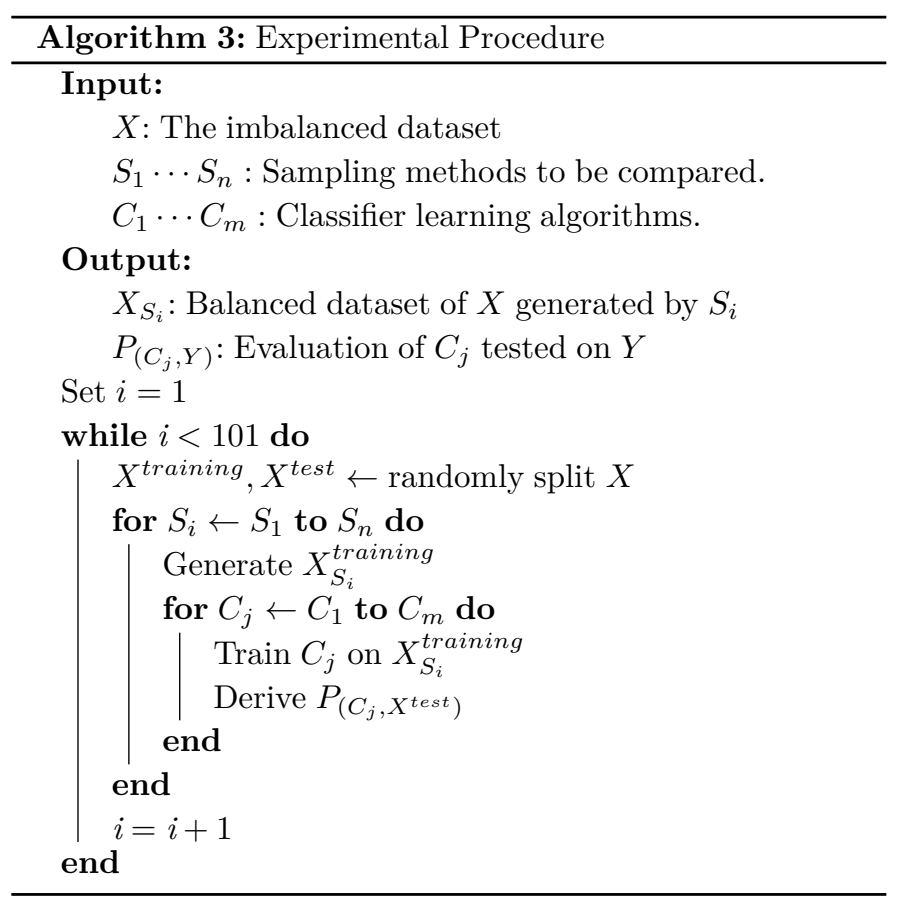

\subsection{Performance Measures}

For performance measures of binary classification, the predicted class labels were compared to the true classes of test data. However, when using such threshold metrics, the class distribution must be carefully considered. The ordinal evaluation methods, such as accuracy, are no longer proper measures for imbalanced datasets because accuracy masks poor classification performance in minority classes, which might lead to wrong conclusions [35], [36], [37], [38], [39], [40].

Other alternatives include the recall, precision, $F_{1}$ score, and G-mean. The majority class is labeled as negatives, 
whereas the minority instances belong to the positive class [35], [36], [39]. Recall (true-positive rates) indicates how many actual minority instances were correctly predicted. Precision represents the prediction accuracy for the minority class. The $F_{1}$ score is the harmonic mean of the recall and precision. The $G$-mean of the true positive and true negative rates is used to use information from both classes. There might be no instances predicted as positive due to skewed class distribution. Though precision and the $F_{1}$ score cannot be defined in such extreme cases, we treat them as zero for a fair comparison. Additionally, we use the area under the receiver operating characteristic (ROC) curve (AUC) value [41], [42]. As the AUC is not sensitive to the distribution between the positive and negative class samples, it is suitable for comparing the performance of different classifiers [42].

To determine whether the differences in mean measures of $R \& R$ and the others are significant, we used the resampled paired $t$ test, which is a popular metric in the machine learning literature [43]. Based on significant paired $t$ statistics, we counted the number of wins and losses between the sampling methods in a pairwise manner. A win indicates the frequency at which each result was recorded as significantly more accurate than for the other algorithms, and a loss indicates the opposite. Win and loss tables are presented in the supplementary materials.

\section{Results and Discussion}

In this section, we present the experimental results of $R \& R$ and other methods on 16 real-world datasets. The results are discussed separately for each dataset type: categorical data, continuous data, and mixed data. For each type, we computed the Monte Carlo means of three performance measures ( $G$-mean, $F_{1}$ score, and AUC) concerning three classifiers (SVM, RF, and NB) across seven sampling strategies (ROSE, SMOTE, ADASYN, MWMOTE, ROS, R\&R, and R\&R with variable selection). Moreover, we provide box plots (Figs. 2, 3 and 4) for each dataset type to demonstrate the distribution of performance measures.

There are some common, remarkable results. First, R\&R outperforms other methods when the dataset has all or some categorical features. This result might be related to the weakness of the other techniques in handling categorical variables, which are ignored when they were first studied. Instead, the raking process in $\mathrm{R} \& \mathrm{R}$ is easily applicable to both categorical and continuous variables. Because almost every dataset in the real world contains categorical variables, this is an advantage of the R\&R sampling method. More detailed characteristics of the results for each data type are handled in the following sections.

Second, a feature selection technique in $R \& R$ could be highly recommended because variable selection generally produces better results by effectively filtering out noisy attributes. Though these improvements are trivial in some datasets, they could be useful in reducing the computation time. When the dataset has numerous variables, reducing the number of attributes to rake could ease the burden of the raking process for generating balanced datasets (Table 2).

Third, the performance of $\mathrm{R} \& \mathrm{R}$ is outstanding when the NB classifier is used as the base classifier or when the $G$-mean is used as an evaluation measure. This tendency becomes
TABLE 2: The number of variables used in $R \& R$ and $R \& R$ with variable selection

\begin{tabular}{|c|c|c|}
\hline \multicolumn{3}{|c|}{ Categorical Datasets } \\
\hline Datasets & R\&R & R\&R $_{v s}$ \\
\hline Boundary & 175 & 22 \\
CaM & 132 & 132 \\
Car good & 6 & 4 \\
Led & 7 & 5 \\
PhosS & 480 & 169 \\
\hline
\end{tabular}

\begin{tabular}{|c|c|c|}
\hline \multicolumn{3}{|c|}{ Continuous Datasets } \\
\hline Datasets & $\mathrm{R} \& \mathrm{R}$ & ${\mathrm{R} \& \mathrm{R}_{v s}}$ \\
\hline CompuStat & 20 & 11 \\
Glass4 & 9 & 3 \\
Mammography & 6 & 6 \\
Oil & 46 & 10 \\
Wine Quality & 11 & 6 \\
Yeast & 8 & 4 \\
\hline
\end{tabular}

\begin{tabular}{|c|c|c|}
\hline \multicolumn{3}{|c|}{ Mixed Datasets } \\
\hline Datasets & $\mathrm{R} \& \mathrm{R}$ & $\mathrm{R \& R}_{v s}$ \\
\hline Abalone & 8 & 8 \\
Flare-F & 11 & 7 \\
Hypo & 19 & 5 \\
Sick & 21 & 10 \\
Vowel0 & 12 & 6 \\
\hline
\end{tabular}

stronger if datasets contain categorical variables. For the G-mean measurement, R\&R obtains higher scores, and the interquartile size of the $R \& R$ box plot is smaller than the other sampling methods, which indicates that the result is stable for the $G$-mean measurement.

Lastly, R\&R is relatively robust and easy to use. It only has parameters for the balance ratio and the number of raking processes. The successful completion of the $R \& R$ algorithm does not depend on these parameters, so the method is more stable across any dataset. For example, MWMOTE must determine six parameters, and one of them is parameter $k_{1}$ for detecting noisy minority samples. If $k_{1}$ is too small, it would assume the sample is noise and remove it from the dataset [4], which might erroneously remove every minority class sample as an unintended consequence. For example, in this experiment, the Wine Quality dataset did not work for this reason 55 times with MWMOTE among the 100 iterations.

\subsection{Categorical Datasets}

First, R\&R performs better than any other method regarding the $G$-mean and AUC measurements regardless of the classifier, which means that $\mathrm{R} \& \mathrm{R}$ has the greatest median value of the G-mean and AUC, at 0.76, among other performance measures. Other comparative methods have a different ranking of performance measures across classifiers. The most significant differences occur in the median values of G-mean and AUC between R\&R and MWMOTE, which has a minimum value for SVM and NB. Moreover, ROSE has a median value for the $G$-mean and AUC similar to SVM, but it has poor performance for RF and NB. Further, SMOTE, ADASYN, and ROS have comparable distributions of the G-mean and 

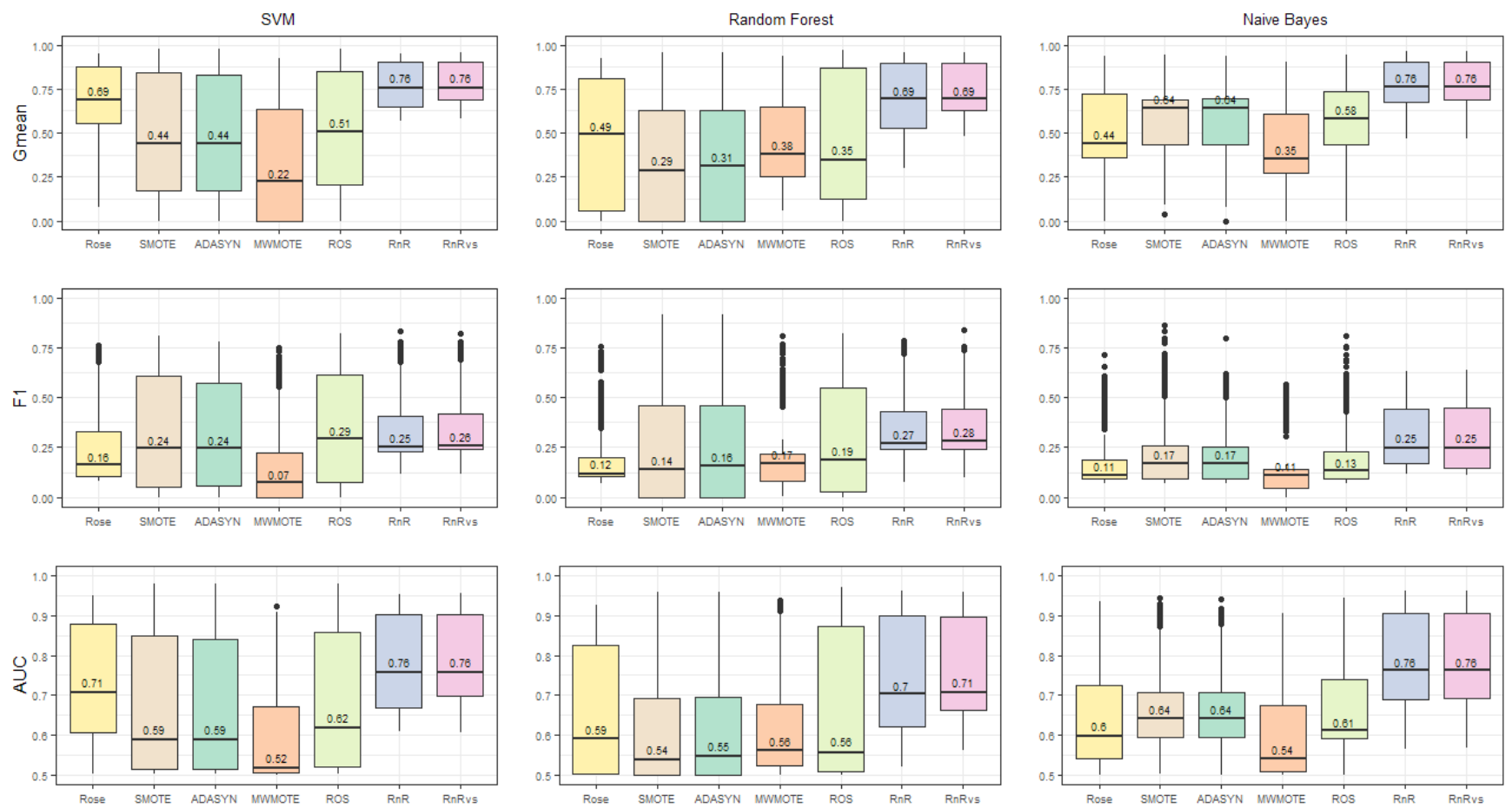

Fig. 2: Box plots of Categorical datasets for each classifiers
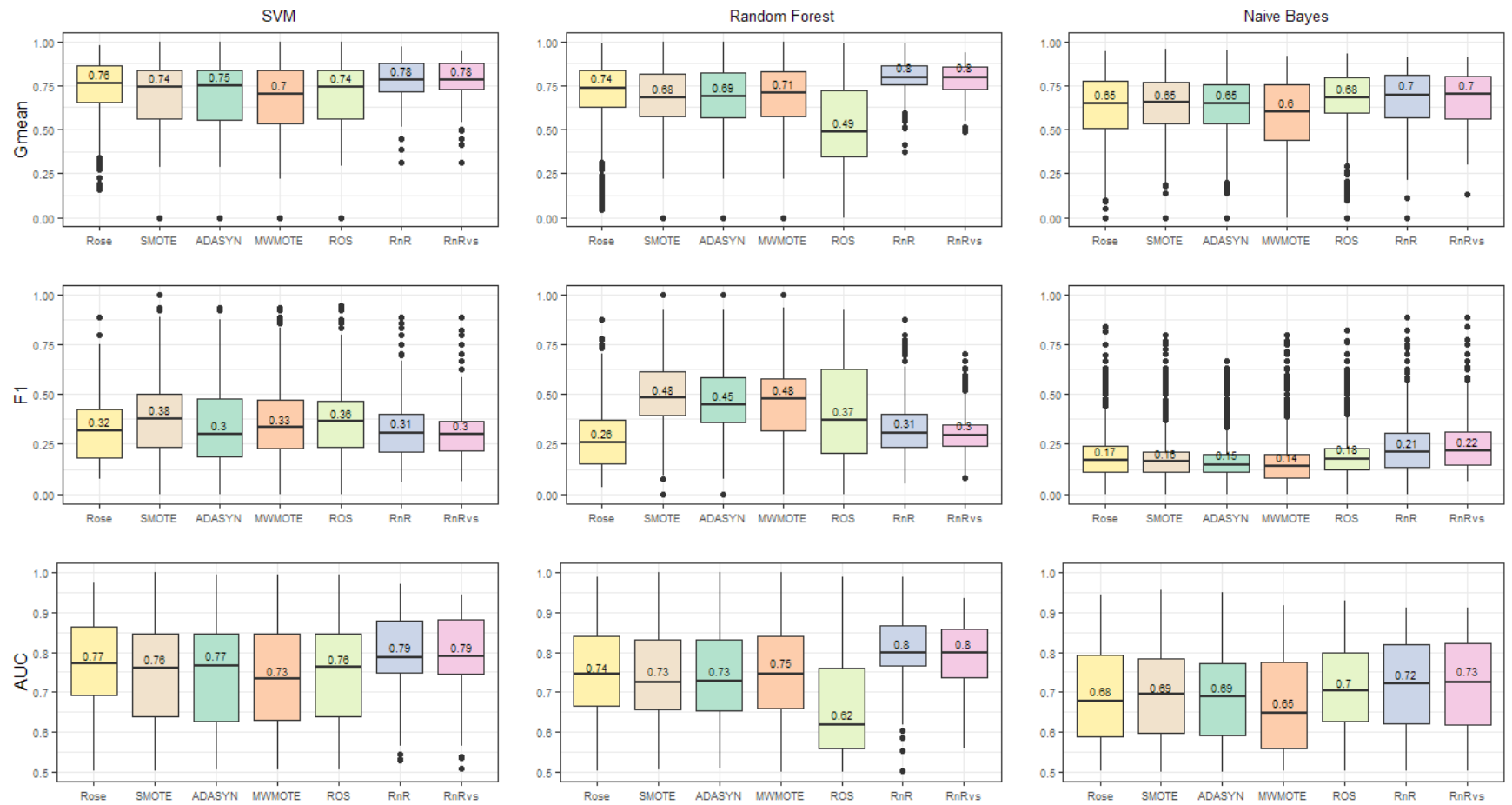

Fig. 3: Box plots of Continuous datasets for each classifiers

AUC values for all three classifiers. Furthermore, R\&R has the smallest interquartile size of $G$-mean for all three classifiers and has an interquartile size of AUC for all three classifiers comparable to those of other methods, which indicates that $\mathrm{R} \& \mathrm{R}$ is more stable than other methods for different scenarios.

Second, for the $F_{1}$ score, the proposed method of R\&R still has the best performance when using RF and NB as the base classifiers. In addition, ROSE has the poorest performance value but seems to be the most robust compared to other methods. When we used the SVM as a base classifier, R\&R had a comparable median value of 0.25 to that of ROS, which had the largest median value at 0.29 . However, the 
interquartile size of ROS is more than double that of $R \& R$, which indicates that the difference between the two methods is not significantly strong.

Third, the results highly recommend using a variable selection process to obtain a smaller classification error. Although we did not use all the variable information in $R \& R$ with the variable selection algorithm, the performance results demonstrate a few differences from $R \& R$, where every attribute was used in the raking procedure. For example, the PhosS dataset used only about one-third of the variables, as depicted in 2 , but the result reveals that variable selection improves performance, especially when SVM or RF is used as a base classifier. Overall, Fig. 2 illustrates that the median value is higher when the raking variables are selected for the SVM $(0.26>0.25)$ and $\operatorname{RF}(0.28>0.27)$ classifiers with the $F_{1}$ score. Moreover, the interquartile size of the performance measures usually decreases, as the AUC indicates.

Lastly, SMOTE and ADASYN tend to create similar results, which might be because ADASYN is one of the most representative extensions of SMOTE, and both approaches employ the KNN-based interpolation. As [4], [35] alleged, this interpolation may duplicate or create incorrect synthetic minority class examples from dense clusters. Therefore, the two algorithms have difficulty in prediction, especially when $\mathrm{RF}$ is used as a classifier.

\subsection{Continuous Datasets}

Initially, three performance measures of seven sampling methods are similar to each other when SVM and NB are used as base classifiers. Different sampling methods with SVM and NB make little difference in the median values of the $G$-mean, $F_{1}$ score, and AUC, but distinct sampling methods with RF create notable results. The seven sampling methods have similar results because SMOTE and its variations were initially well established for continuous data. The remarkable point is that the proposed method has comparable performance to variations of SMOTE for continuous data, despite not being developed only for continuous data.

First, the $G$-mean and AUC results using SVM and NB as classifiers demonstrate that $\mathrm{R} \& \mathrm{R}$ has performance measures comparable to other methods. The median values of performance measures are slightly larger than those of other methods except the $F_{1}$ score with SVM. In addition, R\&R with SVM has a moderately smaller median of the $F_{1}$ score than other methods. However, these differences are not statistically significant, and the $\mathrm{R} \& \mathrm{R}$ with variable selection for the raking procedure has median values similar to those of $R \& R$.

The methods of $\mathrm{R} \& \mathrm{R}$ and $\mathrm{R} \& \mathrm{R}$ with variable selection have an interquartile size of the $G$-mean, $F_{1}$ score, and AUC using SVM as a classifier, especially smaller than all the others. This result indicates that $R \& R$ is more robust to different datasets than other sampling methods. Furthermore, for each dataset, $R \& R$ has stable performance measures across the classifiers. Unlike other methods, $\mathrm{R} \& \mathrm{R}$ has comparable $G$ mean values with different classifiers.

Second, the $G$-mean and AUC results using RF as a classifier demonstrate that $\mathrm{R} \& \mathrm{R}$ has moderately better performance than other methods. The median values of the two measures of $R \& R$ are greater than 0.8 , but the values of the other methods are around 0.7. Moreover, a significant difference exists in the median values of the $G$-mean and AUC between ROS and R\&R. However, the situation changes for the $F_{1}$ score. In addition, R\&R has slightly smaller median values of the $F_{1}$ score than other methods, except for ROSE. Further, SMOTE, ADASYN, and MWMOTE have the highest median $F_{1}$ score, ROS has the second-highest, $\mathrm{R} \& \mathrm{R}$ and $\mathrm{R} \& \mathrm{R}$ with variable section have the third, and ROSE has the minimum median $F_{1}$ score.

A high $G$-mean and low $F_{1}$ score for $\mathrm{R} \& \mathrm{R}$ indicates that it has a high true positive rate but low precision and overpredicts the number of minority samples. One of the reasons for this is that relabeling the majority samples as minority samples occurs when the samples in two classes do not sufficiently overlap. In most imbalanced datasets, it is more important to catch minority instances than to predict the majority class. In other words, the cost concerning the prediction error is different according to the labels. It would be much riskier to have higher costs when the model falsely predicts minority samples as majority samples. In that sense, lower precision would not imply a serious defect. It would be more appropriate for some real-world situations, such as detecting someone dangerous in very rare cases unless the individual was not investigated prior.

Lastly, when R\&R scores high, ROSE also obtains good results, and vice versa. For example, in the case of the SVM and $G$-mean, the three best algorithms were $\mathrm{R} \& \mathrm{R}, \mathrm{R} \& \mathrm{R}$ with variable selection, and ROSE $(0.78,0.78$, and 0.76 , respectively). In comparison, these were the three worst algorithms when RF classifiers and the $F_{1}$ score were used $(0.33,0.31$, and 0.26 , respectively). In other words, R\&R and ROSE tend to move together because if a relabeled majority sample in $R \& R$ is one of the neighbors of each minority sample in the overlapped area, it has an effect similar to synthetic samples generated using the estimated kernel density for each minority sample.

\subsection{Mixed Datasets}

The results of mixed datasets are somewhat in the intermediate state between those of the categorical and continuous datasets. First, as the results of the categorical datasets demonstrated, R\&R outperforms every other method regarding the $G$-mean and AUC measurements for all three classifiers. In addition, $\mathrm{R} \& \mathrm{R}$ has a median value of the $G$ mean and AUC from 0.78 to 0.87 , whereas others have 0.79 at most. Furthermore, while R\&R always has the greatest median value of two measurements for each classifier, other comparative methods have a different performance ranking across the classifiers. For example, ROSE takes 0.79 as the median value of AUC and SVM as a base classifier, which is the next greater value after $R \& R(0.87)$ and $R \& R$ with variable selection (0.86). However, when we used RF as a classifier, the performance of ROSE became the worst, which indicates that other sampling methods depend on its performance on the classifiers.

Second, the most significant differences in the median values of measurements are between R\&R and ROSE. Although the difference becomes smaller when the SVM was used as a classifier, ROSE was beaten by every other method, and R\&R overwhelmed the others, including ROSE. This difference becomes extreme when the G-mean is used as an evaluation 

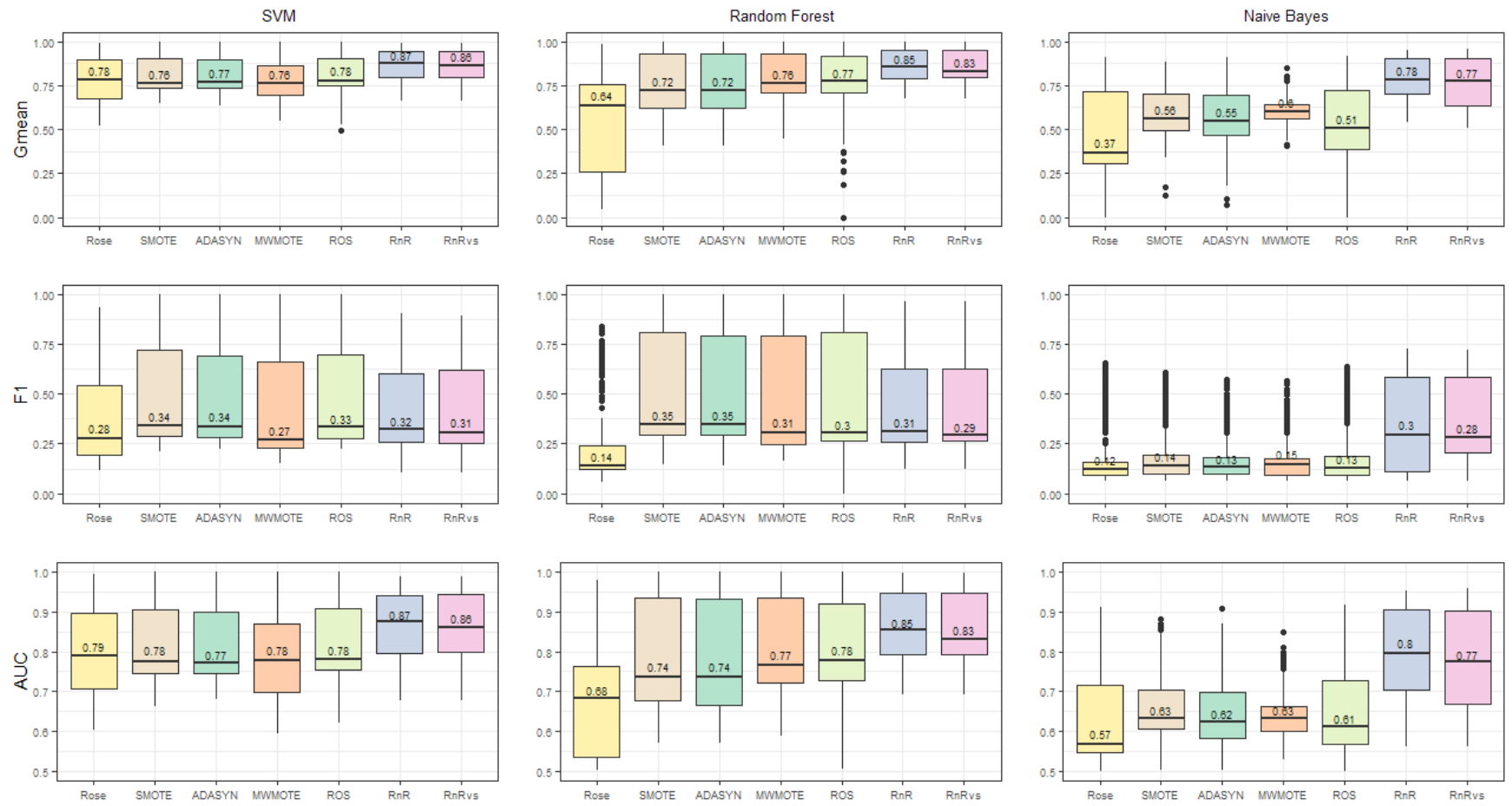

Fig. 4: Box plots of Mixed datasets for each classifiers

measurement and when NB is employed as a base classifier, 0.41. Furthermore, as the box plots of ROSE are below the others, and its interquartile size is large, we conclude that ROSE has a problem handling the mixed type of datasets.

Third, the integration of $\mathrm{R} \& \mathrm{R}$ with the $\mathrm{NB}$ classifier stands out. Although $R \& R$ has a larger interquartile size, the third quantile of $\mathrm{R} \& \mathrm{R}$ is still higher than most other sampling methods' first quartile. For example, though its variation might be greater than the others, the median measurements ( $G$-mean and AUC) of the R\&R algorithm (0.78 and 0.80, respectively) are much higher than all others.

Fourth, like the continuous datasets, R\&R had a lower median $F_{1}$ score except with NB. However, we do not need to consider it seriously because the difference is at most 0.04 , which comes from the comparison between $R \& R$ and ADASYN with RF as the classifier. This difference is much smaller compared to the difference for NB when R\&R outperforms all other methods. Moreover, the smaller interquartile size of R\&R in RF could offset its slightly smaller median $F_{1}$ score (0.31).

Lastly, it is intriguing that the variable selection in $R \& R$ could worsen performance. Unlike other data type cases, every performance measure records a lower score when the variable selection was used. When RF was used as a classifier, the measurements of R\&R decreased to about 0.02. However, this point is still not a serious concern compared to other algorithms.

\section{Conclusion}

We propose a new resampling approach that generates balanced samples for binary classification. Three bootstrap samples were generated from raking, relabeling, and resampling techniques. The balanced minority samples consist of two bootstrap samples: one generated from minority samples and the other generated from majority samples. The first bootstrap samples were obtained using the conventional bootstrap technique based on simple random sampling with replacement. The second bootstrap samples were constructed from the proposed $\mathrm{R} \& \mathrm{R}$ algorithm. We obtained raking weights for each unit in the majority sample and constructed bootstrap samples generated from the majority samples with a probability proportional to the raking weights. Those bootstrap samples have sample moments or proportions similar to those of the minority samples. Thus, we can use these as if they were minority samples. We also apply the same technique to construct balanced majority samples, which are the third type of bootstrap samples.

The generated balanced samples are used to learn three classifiers, including SVM, RF, and NB. Afterward, we compared the performance of the proposed resampling method to other popular resampling techniques: ROSE, SMOTE, ADASYN, and MWMOTE using three metrics, the $G$-mean, $F_{1}$ score, and AUC. The proposed R\&R algorithm generally works well across all experimental setups and is less sensitive to data types and classifiers. Moreover, the proposed $\mathrm{R} \& \mathrm{R}$ algorithm is more desirable considering that it does not require a distribution estimation procedure and is easy to implement even for large, complicated, and high-dimensional data. All datastes used in this paper were uploaded to https://github.com/dataintegration38/RnR-dataset.

\section{Acknowledgment}

S. Park's and J. Im's are supported by the National Research Foundation (NRF) Korea, NRF-2019R1G1A1002232 and NRF-2021R1C1C1014407, respectively. 


\section{References}

[1] H. He and E. A. Garcia, "Learning from imbalanced data," IEEE Transactions on knowledge and data engineering, vol. 21, no. 9, pp. 1263-1284, 2009.

[2] I. Nekooeimehr and S. K. Lai-Yuen, "Adaptive semiunsupervised weighted oversampling (a-suwo) for imbalanced datasets," Expert Systems with Applications, vol. 46, pp. 405416, 2016.

[3] N. V. Chawla, "Data mining for imbalanced datasets: An overview," Data mining and knowledge discovery handbook, pp. 875-886, 2009.

[4] S. Barua, M. M. Islam, X. Yao, and K. Murase, "Mwmotemajority weighted minority oversampling technique for imbalanced data set learning," IEEE Transactions on knowledge and data engineering, vol. 26, pp. 405-425, 2012.

[5] J. Mathew, C. K. Pang, M. Luo, and W. H. Leong, "Classification of imbalanced data by oversampling in kernel space of support vector machines," IEEE transactions on neural networks and learning systems, vol. 29, no. 9, pp. 4065-4076, 2017.

[6] N. V. Chawla, K. W. Bowyer, L. O. Hall, and W. P. Kegelmeyer, "SMOTE: Synthetic minority over-sampling technique," Journal of Artificial Intelligence Research, vol. 16, pp. 321-357, 2002.

[7] A. Fernández, S. García, F. Herrera, and N. Chawla, "Smote for learning from imbalanced data: Progress and challenges, marking the 15-year anniversary," J. Artif. Intell. Res., vol. 61, pp. 863-905, 2018.

[8] H. He, Y. Bai, E. A. Garcia, and S. Li, "ADASYN: Adaptive synthetic sampling approach for imbalanced learning," 2008 IEEE International Joint Conference on Neural Networks (IEEE World Congress on Computational Intelligence), vol. 16, pp. 1322-1328, 2008.

[9] G. Cohen, M. Hilario, H. Sax, S. Hugonnet, and A. Geissbuhler, "Learning from imbalanced data in surveillance of nosocomial infection," Artificial intelligence in medicine, vol. 37, no. 1, pp. $7-18,2006$.

[10] H. Han, W. Y. Wang, and B. H. Mao, "Borderline-smote: a new over-sampling method in imbalanced data sets learning," In International conference on intelligent computing (pp. 878-887), pp. 878-887, 2005.

[11] G. Menardi and N. Torelli, "Training and assessing classification rules with imbalanced data," Data mining and knowledge discovery, vol. 28, pp. 92-122, 2014.

[12] C. Bunkhumpornpat, K. Sinapiromsaran, and C. Lursinsap, "Safe-level-smote: Safe-level-synthetic minority over-sampling technique for handling the class imbalanced problem," in Pacific-Asia conference on knowledge discovery and data mining. Springer, 2009, pp. 475-482.

[13] X. T. Dang, D. H. Tran, O. Hirose, and K. Satou, "Spy: A novel resampling method for improving classification performance in imbalanced data," 2015 Seventh International Conference on Knowledge and Systems Engineering (KSE), pp. 280-285, 2015.

[14] L. T. Nghiem, T. T. Thu, and T. T. Nghiem, "Masi: moving to adaptive samples in imbalanced credit card dataset for classification," in 2018 IEEE International Conference on Innovative Research and Development (ICIRD). IEEE, 2018, pp. 1-5.

[15] D. Mease, A. J. Wyner, and A. Buja, "Boosted classification trees and class probability/quantile estimation." Journal of Machine Learning Research, vol. 8, no. 3, 2007.

[16] N. Japkowicz et al., "Learning from imbalanced data sets: a comparison of various strategies," in AAAI workshop on learning from imbalanced data sets, vol. 68. AAAI Press Menlo Park, CA, 2000, pp. 10-15.

[17] Y. Tang, Y.-Q. Zhang, N. V. Chawla, and S. Krasser, "Svms modeling for highly imbalanced classification," IEEE Transactions on Systems, Man, and Cybernetics, Part B (Cybernetics), vol. 39, no. 1, pp. 281-288, 2008.

[18] Z.-H. Zhou and X.-Y. Liu, "Training cost-sensitive neural networks with methods addressing the class imbalance problem," IEEE Transactions on knowledge and data engineering, vol. 18, no. 1, pp. 63-77, 2005.

[19] W. A. Fuller, Sampling Statistics. Hoboken, NJ, USA: John Wiley \& Sons, Inc, 2009.

[20] M. Silvapulle, "On the existence of maximum likelihood estimators for the binomial response models," Journal of the Royal Statistical Society. Series B, vol. 43, pp. 310-313, 1981.

[21] A. B. Owen, "Infinitely imbalanced logistic regression," Journal of Machine Learning Research, vol. 8, pp. 761-773, 2007.
[22] M. Pastore and A. Calcagnì, "Measuring distribution similarities between samples: A distribution-free overlapping index," Frontiers in Psychology, vol. 10, p. 1089, 2019.

[23] J. C. Deville and C. E. Särndal, "Calibration estimators in survey sampling," Journal of the American Statistical Association, vol. 87, pp. 376-382, 1992.

[24] J. Im, Y. Seo, K. S. Cetin, and J. Singh, "Energy efficiency in us residential rental housing: Adoption rates and impact on rent," Applied Energy, vol. 205, pp. 1021-1033, 2017.

[25] J. Alcalá-Fdez and A. Fernandez and J. Luengo and J. Derrac and S. García and L. Sánchez and F. Herrera, "Keel datamining software tool: Data set repository, integration of algorithms and experimental analysis framework," https://sci2s.ugr. es/keel/datasets.php, 2011.

[26] D. Dua and C. Graff, "UCI machine learning repository," 2017. [Online]. Available: http://archive.ics.uci.edu/ml

[27] D. A. Cieslak, T. R. Hoens, N. V. Chawla, and W. P. Kegelmeyer, "Hellinger distance decision tree implementation data sets," https://www3.nd.edu/ dial/hddt/, 2012.

[28] P. Radivojac, N. V. Chawla, A. K. Dunker, and Z. Obradovic, "Classification and knowledge discovery in protein databases," Journal of Biomedical Informatics, vol. 37, no. 4, pp. 224-239, 2004, biomedical Machine Learning. [Online]. Available: https:// www.sciencedirect.com/science/article/pii/S1532046404000760

[29] M. Kubat, R. C. Holte, and S. Matwin, "Machine learning for the detection of oil spills in satellite radar images," Machine Learning, vol. 30, no. 2, pp. 195-215, Feb 1998. [Online]. Available: https://doi.org/10.1023/A:1007452223027

[30] K. S. WOODS, C. C. DOSS, K. W. BOWYER, J. L. SOLKA, C. E. PRIEBE, and W. P. KEGELMEYER, "Comparative evaluation of pattern recognition techniques for detection of microcalcifications in mammography," International Journal of Pattern Recognition and Artificial Intelligence, vol. 07, no. 06, pp. 1417-1436, Dec 1993. [Online]. Available: https: //doi.org/10.1142/S0218001493000698

[31] M. Mohamad, A. Selamat, I. M. Subroto, and O. Krejcar, "Improving the classification performance on imbalanced data sets via new hybrid parameterisation model," Journal of King Saud University - Computer and Information Sciences, 2019.

[32] W. S. Noble, "What is a support vector machine?" Nat Biotechnol 24, pp. 1565-1567, 2006

[33] L. Breiman, A. Cutler, A. Liaw, and M. Wiener, Breiman and Cutler's Random Forests for Classification and Regression, 2018. [Online]. Available: https://cran.r-project.org/web/packages/ randomForest/randomForest.pdf

[34] D. Meyer, E. Dimitriadou, K. Hornik, A. Weingessel, F. Leisch, C.-C. Chang, and C.-C. Lin, Misc Functions of the Department of Statistics, Probability Theory Group (Formerly: E1071), TU Wien, 2021. [Online]. Available: https://cran.r-project.org/web/packages/e1071/e1071.pdf

[35] A. Fernández, S. García, M. Galar, R. C. Prati, B. Krawczyk, and F. Herrera, Learning from Imbalanced Data Sets. the Permissions Department, John Wiley \& Sons, Inc.: Springer, Cham, 2013.

[36] H. HE and Y. MA, IMBALANCED LEARNING Foundations, Algorithms, and Applications. Cham, Switzerland: WileyIEEE, 2018.

[37] O. Maimon and L. Rokach, Data Mining and Knowledge Discovery Handbook. Berlin, Heidelberg: Springer-Verlag, 2005.

[38] G. M. Weiss, "Mining with rarity: A unifying framework," SIGKDD Explor. Newsl., vol. 6, no. 1, p. 7-19, Jun. 2004. [Online]. Available: https://doi.org/10.1145/1007730.1007734

[39] H. He and E. A. Garcia, "Learning from imbalanced data," IEEE Transactions on Knowledge and Data Engineering, vol. 21, no. 9, pp. 1263-1284, 2009.

[40] Yanminsun, A. Wong, and M. S. Kamel, "Classification of imbalanced data: a review," International Journal of Pattern Recognition and Artificial Intelligence, vol. 23, 112011.

[41] T. Fawcett, "Roc graphs: Notes and practical considerations for researchers," Machine Learning, vol. 31, pp. 1-38, 012004.

[42] — , "An introduction to roc analysis," Pattern Recognition Letters, vol. 27, no. 8, pp. 861-874, 2006. [Online]. Available: https://www.sciencedirect.com/science/article/pii/ S016786550500303X

[43] T. G. Dietterich, "Approximate statistical tests for comparing supervised classification learning algorithms," Neural Computation, vol. 10, no. 7, pp. 1895-1923, 1998. 


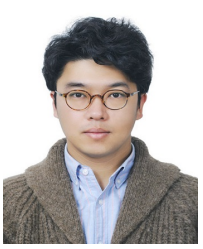

Seunghwan Park received the $\mathrm{PhD}$ degree in statistics from Seoul National University in 2013.

$\mathrm{He}$ is currently an associate professor of the department of Informationand statistics at Kangwon National University. His research interests include survey sampling, missing data analysis, small area estimation and causal inference.

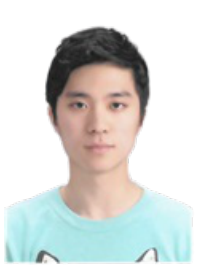

Hae-Hwan Lee received the Bachelor's degree in econoimcs and statistics from Yonsei University in 2020. He is currently a master candidate of the department of statistics \& data science at Yonsei University. His research interests include data mining, pattern recognition and data privacy.

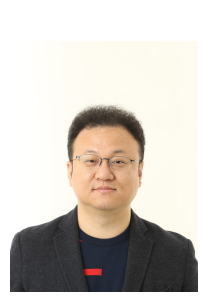

Jongho $\mathrm{Im}$ received the $\mathrm{PhD}$ degree in statistics from lowa State University in 2015 . He is currently an associate professor of the department of statistics \& data science at Yonsei University. $\mathrm{His}$ research interests include data-driven science, business intelligence, computational statistics, data privacy, missing data analysis, and text data analysis. 